\title{
Plasma Osteopontin Level in Chronic Liver Disease and Hepatocellular Carcinoma
}

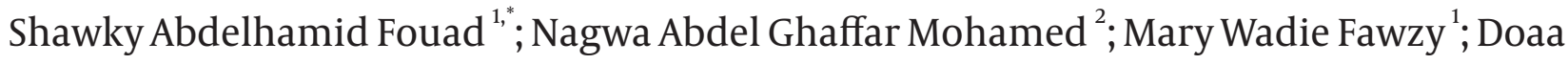 \\ Ali Moustafa ${ }^{1}$ \\ ${ }^{1}$ Department of Internal Medicine, Faculty of Medicine, Cairo University, Cairo, Egypt \\ ${ }^{2}$ Department of Clinical and Chemical Pathology, National Research Center, Giza, Egypt \\ ${ }^{*}$ Corresponding Author: Shawky Abdelhamid Fouad, Department of Internal Medicine, Faculty of Medicine, Cairo University, Cairo, Egypt. Tel: $+20-01223658902$, \\ E-mail: shawkyabdelhamid@gmail.com
}

Received: June 14, 2015; Revised: July 31, 2015; Accepted: August 16, 2015

\begin{abstract}
Background: Osteopontin (OPN) is a secreted glycoprotein and is frequently associated with various tumors.
Objectives:We sought to investigate the clinical usefulness of the level of plasma OPN, compared to $\alpha$-fetoprotein (AFP), as a biomarker for hepatocellular carcinoma (HCC) and to evaluate its diagnostic value in nonalcoholic fatty liver disease (NAFLD) and its relationship with clinical and laboratory features of HCC and NAFLD.

Patients and Methods: The study was performed on 120 subjects classified into 5 groups: Group I included 25 chronic non-cirrhotic hepatitis C virus (HCV)-infected patients; Group II encompassed 25 patients with chronic HCV infection with liver cirrhosis; Group III comprised 25 patients with chronic HCV with liver cirrhosis and HCC; Group IV was comprised of 25 patients with NAFLD; and Group V consisted of 20 healthy age- and sex-matched controls. All the participants were subjected to history taking and clinical and abdominal ultrasonographic examinations as well as the following laboratory investigations: liver function tests, complete blood count, blood sugar, hepatitis B surface antigen, hepatitis C virus antibodies, HCV-RNA by qualitative polymerase chain reaction (for Groups I, II, and III) and serum AFP and plasma OPN levels.

Results: There were statistically significant differences in plasma OPN levels between the HCC group (401 $\pm 72 \mathrm{ng} / \mathrm{mL}$ ) and the other groups, between the cirrhotic group ( $258.3 \pm 35 \mathrm{ng} / \mathrm{mL}$ ) and the non-cirrhotic group (HCV group, $168.7 \pm 41 \mathrm{ng} / \mathrm{mL}$; fatty liver group, 106.7 $\pm 35 \mathrm{ng} / \mathrm{mL}$ ), and between the chronic non-cirrhotic HCV group and the fatty liver group (I and IV) and the controls ( $35.1 \pm 6 \mathrm{ng} / \mathrm{mL}$ ). In the HCC group, the diagnostic value of OPN was comparable to that of AFP at a cutoff value of $280 \mathrm{ng} / \mathrm{mL}$, achieving sensitivity, specificity, and overall accuracy of $100 \%, 98 \%$, and $96 \%$, respectively. Regarding the validity of plasma OPN as a predictor of fatty change, our results revealed a diagnostic accuracy of $50 \%$ with $70 \%$ sensitivity, $45 \%$ specificity, $50 \%$ positive predictive value, and $75 \%$ negative predictive value at a cutoff value of $134 \mathrm{ng} / \mathrm{mL}$.

Conclusions: Plasma OPN is comparable to AFP as a diagnostic marker and is related to the severity of liver involvement in HCC patients. Plasma OPN is of diagnostic potential value in NAFLD.
\end{abstract}

Keywords: Osteopontin; Hepatocellular Carcinoma; Fatty Liver

\section{Background}

Osteopontin (OPN) is a multifunctional protein, involved in pathological conditions such as inflammation, immunity, angiogenesis, fibrosis, and cancer progression in various tissues (1). OPN, also known as early T-cell activation gene-1, is a secreted phosphoprotein 1 and has been implicated in the pathogenesis of various inflammatory and fibrotic disorders. It stimulates T-cell proliferation and induces $T$ cells and macrophages to express other $\mathrm{T}$ helper type 1 cytokines during inflammation (2). OPN also induces the accumulation of extracellular matrix by binding to type I collagen, fibronectin, and osteocalcin, thereby contributing to tissue fibrosis (3). OPN comprises multiple functional domains, with a high sialic acid content, an aspartate-rich domain, a calcium-binding

domain, a thrombin cleavage site, many residues with consensus for phosphorylation, and an integrin-binding arginine-glycine-aspartate motif, which play a key role in several inflammatory disorders (4). High levels of circulating OPN have been demonstrated in patients with hepatitis $\mathrm{C}$ virus (HCV) infection (5) as well as gastric and liver cancers (6). Nonalcoholic steatohepatitis (NASH) is a leading cause of cirrhosis. Syn et al. (7) showed that NASH-related cirrhosis is associated with the Hedgehog pathway activation. The gene-encoding OPN, a profibrogenic extracellular matrix protein and cytokine, is a direct transcriptional target of the Hedgehog pathway (7).

$\mathrm{HCV}$ is a major cause of post-transfusion hepatitis and liver transplantation in many countries (8). Liver-related 
Fouad SA et al.

complications such as cirrhosis and hepatocellular carcinoma (HCC) are among the major complications in HCVinfected patients (9).

HCC is a highly aggressive carcinoma of the liver; it is the fifth most common cancer worldwide and the third leading cause of cancer-related death. Risk factors for HCC include infection with hepatitis B virus (HBV) or $\mathrm{HCV}$, alcoholic cirrhosis, and exposure to environmental toxins such as aflatoxins (10). Chronic hepatitis $C$ is the most common cause of chronic liver disease and cirrhosis; it is also the most common indication for liver transplantation in the United States (US), Australia, and most of Europe (11). The Egyptian Demographic Health Survey (EDHS), a cross-sectional survey including HCV biomarkers, was conducted in 2008 on a large nationally representative sample. It estimated that the HCV prevalence was $14.7 \%$ among individuals between 15 and 59 years of age (12). Accordingly, Egypt has the highest HCV prevalence in the world (13).

\section{Objectives}

In this study, we aimed to assess the value of plasma OPN as a biomarker for HCC and to evaluate its diagnostic value in nonalcoholic fatty liver disease (NAFLD) and its relationship with clinical and laboratory features of HCC and NAFLD.

\section{Patients and Methods}

\subsection{Subjects}

This study was carried out in the Department of Internal Medicine, Faculty of Medicine, Cairo University, Cairo, Egypt, between May 2014 and March 2015, and included 120 subjects subdivided into 5 groups: Group I comprised 25 patients with HCV without cirrhosis; Group II included 25 patients with HCV with liver cirrhosis; Group III included 25 patients with HCC on top of cirrhosis; Group IV was comprised of 25 patients with NAFLD; and Group V consisted of 20 adult healthy age- and sex-matched control subjects. Written informed consents were obtained from all the participants.

\subsection{Exclusion Criteria}

The exclusion criteria were comprised of HBV and HCV coinfection; any malignant diseases other than HCC; diabetes mellitus; autoimmune or inflammatory conditions; respiratory, cardiac, or renal troubles; and pregnancy.

\subsection{Methods}

All the participants were subjected to full history taking, abdominal ultrasonography, and laboratory evaluation comprising complete blood count, blood sugar, bilirubin, aspartate aminotransferase (AST), alanine transaminase (ALT), serum albumin, urea, creatinine, international normalized ratio, hepatitis B surface an- tigen, hepatitis C virus antibodies, HCV-RNA by qualitative polymerase chain reaction (for Groups I, II, and III), serum $\alpha$-fetoprotein (AFP), and plasma OPN levels. Five $\mathrm{mL}$ of fasting ( 6 - 8 hour) venous blood samples was collected from each subject participating in the study and divided into 2 parts: The first part, $3 \mathrm{~mL}$ of blood, was put in a plain tube and left to clot, and the serum was separated by centrifugation for 15 minute at $1,000 \times$ g to determine the liver and kidney functions and AFP. The second part, $2 \mathrm{~mL}$ of venous blood, was collected on ethylene diamine tetraacetate (EDTA) and centrifuged for 15 minute at 1,000 $\times \mathrm{g}$ within 30 minute of collection and stored at $-20^{\circ} \mathrm{C}$ to determine plasma OPN. Complete blood count was performed on a Coulter Counter T890 (Coulter Counter, Harpenden, UK). Liver function tests (serum bilirubin, ALT, AST, and albumin) were determined calorimetrically on a Hitachi 912 AutoAnalyzer (Hitachi 912, Hitachi, Japan). Prothrombin time and prothrombin concentration were performed using the standard thromboplastin method on an automated blood coagulation analyzer (Siemens AG, Erlangen, Germany). AFP was determined using the enzyme-linked immunosorbent assay (ELISA) kit supplied by Kamiya Biomedical Company (Seattle, US) (14). Plasma OPN was determined using an immunoassay (ELISA) kit supplied by Fisher Scientific UK Ltd (Bishop Meadow Road, Loughborough, UK) (15).

\subsection{Statistical Analysis}

Analysis of data was done using Statistical Package for the Social Sciences (SPSS), version 16, to describe the quantitative variables (mean, standard deviation [SD], and range). Description of the qualitative variables (number and percentage) was carried out using the $\chi^{2}$ test. The one-way analysis of variance (ANOVA) was used to compare more than 2 groups as regards the quantitative variables in parametric data. The Spearman correlation test was utilized for the nonparametric data, while the Pearson correlation test was employed for the parametric measurements. AP $\leq 0.05$ was considered statistically significant. Receiver operating characteristic (ROC) curves were plotted. The area under the ROC curve (AUC) was calculated, and its 95\% confidence interval (CI) was calculated via 1,000 bootstrap samples. Optimal cutoffs were calculated using the maximum sum of sensitivity and specificity and the minimum distance to the top-left corner of the ROC curve.

\section{Results}

The present study recruited 120 subjects comprised of 85 (70.83\%) males and 35 (29.17\%) females. Their ages ranged from 24 to 75 years with a mean age of $49.72 \pm 19.3$ years (Table 1). Apropos clinical manifestations, $44.6 \%$ of our patients had jaundice, $29.23 \%$ had a history of encephalopathy, $36 \%$ had ascites, and $43 \%$ had bilateral lower limb edema. Moreover, $52 \%$ of the patients were anemic and $47 \%$ were thrombocytopenic. Additionally, $49 \%$ of the patients 
had elevated liver enzymes, 44\% had elevated serum bilirubin (biphasic), and $48 \%$ had decreased serum albumin.

A comparison of the demographic data of all the patients and controls in terms of age, sex, and body mass index (BMI) revealed that the fatty liver group had a significantly higher BMI than the other groups $(\mathrm{P}<0.001)$ according to the one-way ANOVA; nevertheless, there were no statistically significant differences between all the groups concerning age and sex. Regarding the results of the clinical parameters, there were statistically significant differences between all the groups vis-a-vis jaundice, encephalopathy, ascites, and lower limb ede$\mathrm{ma}(\mathrm{P}<0.001)$. Concerning the results of the laboratory parameters, there were statistically significant differences in ALT and AST levels between the control group and the other groups $(\mathrm{P}<0.001)$. There was a statistically significant difference in serum bilirubin between the non-cirrhotic groups (Groups I, IV, and V) and the cirrhotic (Group II) and HCC (Group III) groups. Also, there were statistically significant differences in serum albumin and prothrombin time between the control and non-cirrhotic groups and the cirrhotic and HCC groups. Furthermore, there were statistically significant differences in the platelet count and the hemoglobin concentration between the cirrhotic and HCC groups and the other groups $(\mathrm{P}<0.001)$ (Table 1$)$.

In regard to the correlation between the OSP level and the different variables in the different groups, our results revealed only significant correlations between OSP and the degree of lower limb edema in Group II ( $\mathrm{r}=0.39 ; \mathrm{P}=$ $0.03)$ and between OSP and ALT $(r=0.56 ; \mathrm{P}=0.02)$, bilirubin $(\mathrm{r}=0.025 ; \mathrm{P}=0.01)$, and AFP in Group III $(\mathrm{r}=-0.45 ; \mathrm{P}=$ 0.03) (Table 2 and Figure 1 ).

Our results also demonstrated that AFP, bilirubin, and lower limb edema were directly correlated with the OPN level according to the stepwise technique $(\mathrm{P}<0.05)$. On the other hand regarding the correlation between the AFP level and the different variables in the different groups, our results revealed only significant correlations between AFP and AST in Group II $(r=0.56 ; \mathrm{P}=0.02)$ and between AFP and the international normalized ratio in Group III $(r=-0.43 ; P=0.02)$.

\begin{tabular}{|c|c|c|c|c|c|}
\hline Variables & Group I & Group II & Group III & Group IV & Group V \\
\hline Age, $y^{c}$ & $47 \pm 11$ & $53 \pm 7$ & $49.8 \pm 8$ & $48 \pm 10$ & $48 \pm 10$ \\
\hline \multicolumn{6}{|l|}{ Gender $^{c}$} \\
\hline Male & $19(76)$ & $18(72)$ & $18(72)$ & $16(64)$ & $14(70)$ \\
\hline Female & $6(24)$ & $7(28)$ & $7(28)$ & $9(36)$ & $6(30)$ \\
\hline $\mathrm{BMI}^{\mathrm{d}}, \mathrm{kg} / \mathrm{m}^{2}$ & $26 \pm 8$ & $23.6 \pm 7$ & $25.5 \pm 11$ & $39 \pm 10$ & $29 \pm 2.8$ \\
\hline ALT, IU/L & $44.2 \pm 5$ & $35 \pm 10$ & $31.9 \pm 5$ & $48.5 \pm 6$ & $23 \pm 4.9^{\mathrm{e}}$ \\
\hline AST, IU/L & $46 \pm 21$ & $41 \pm 17$ & $28 \pm 10$ & $40 \pm 6$ & $16.4 \pm 3.5^{\mathrm{e}}$ \\
\hline Bilirubin, mg/dL ${ }^{f}$ & $0.94 \pm 0.3$ & $2 \pm 0.9$ & $3.2 \pm 0.4$ & $1.16 \pm 0.2$ & $0.95 \pm 0.1$ \\
\hline Albumin, g/dL $\mathbf{g}$ & $4.5 \pm 2$ & $3.2 \pm 1.6$ & $2.6 \pm 0.4$ & $4.1 \pm 0.3$ & $4 \pm 0.2$ \\
\hline PT, sec $\mathrm{g}$ & $12.3 \pm 4$ & $16.7 \pm 5$ & $18 \pm 3$ & $11 \pm 2.3$ & $10.8 \pm 2$ \\
\hline $\mathbf{P C}, \% \mathbf{f}$ & $90 \pm 11$ & $50 \pm 12$ & $41.2 \pm 10$ & $93 \pm 12$ & $99 \pm 10$ \\
\hline INR $^{f}$ & $1.03 \pm 0.4$ & $1.8 \pm 0.6$ & $2.1 \pm 0.2$ & $1.06 \pm 0.3$ & $1 \pm 0.3$ \\
\hline Platelets, $\times 10^{9} / \mathrm{L}$ & $209 \pm 50$ & $135 \pm 50$ & $120 \pm 40$ & $234 \pm 80$ & $224 \pm 67$ \\
\hline HB, g/dL $\mathbf{g}$ & $14 \pm 2$ & $11 \pm 2.2$ & $10 \pm 0.9$ & $11.8 \pm 1.4$ & $14 \pm 1.4$ \\
\hline AFP, ng/mL & $4.1 \pm 1.8$ & $3.9 \pm 1.3$ & $771 \pm 165^{e}$ & $6.8 \pm 4$ & $8.7 \pm 7$ \\
\hline \multicolumn{6}{|c|}{$\begin{array}{l}\text { abbreviations: AFP, } \alpha \text {-fetoprotein; ALT, alanine aminotransferas } \\
\text { normalized ratio; HB, hemoglobin; PC, prothrombin concentration; I } \\
\text { b Data are presented as mean } \pm \text { standard deviation or number (\%). } \\
\text { c There were no significant differences between all groups. } \\
\text { d There were no significant differences between group I, III and V. } \\
\text { e Statistically significant. } \\
\text { f There were no significant differences between group I, IV and V. } \\
\text { g There were no significant differences between group I, IV and V and }\end{array}$} \\
\hline
\end{tabular}


Fouad SA et al.

\begin{tabular}{|c|c|c|c|c|c|c|c|c|c|c|}
\hline \multirow[t]{2}{*}{ Variables } & \multicolumn{2}{|c|}{ Group I } & \multicolumn{2}{|c|}{ Group II } & \multicolumn{2}{|c|}{ Group III } & \multicolumn{2}{|c|}{ Group IV } & \multicolumn{2}{|c|}{ Group V } \\
\hline & $\mathbf{r}$ & $\mathbf{P}$ & $\mathbf{r}$ & $\mathbf{P}$ & $\mathbf{r}$ & $\mathbf{P}$ & $\mathbf{r}$ & $\mathbf{P}$ & $\mathbf{r}$ & $\mathbf{P}$ \\
\hline ALT & 0.09 & 0.12 & 0.02 & 0.56 & 0.56 & 0.02 & 0.16 & 0.26 & 0.10 & 0.20 \\
\hline AST & 0.11 & 0.24 & 0.15 & 0.55 & 0.18 & 0.20 & 0.06 & 0.70 & 0.04 & 0.66 \\
\hline Bilirubin & -0.03 & 0.55 & -0.07 & 0.40 & 0.25 & 0.01 & 0.15 & 0.34 & 0.19 & 0.30 \\
\hline Albumin & 0.13 & 0.25 & -0.11 & 0.20 & -0.14 & 0.18 & 0.11 & 0.22 & 0.01 & 0.92 \\
\hline PT & 0.14 & 0.19 & 0.17 & 0.39 & 0.12 & 0.33 & -0.08 & 0.67 & -0.02 & 0.87 \\
\hline PC & 0.23 & 0.11 & 0.23 & 0.11 & 0.20 & 0.16 & 0.18 & 0.26 & -0.19 & 0.29 \\
\hline INR & 0.16 & 0.33 & 0.10 & 0.30 & 0.18 & 0.19 & 0.10 & 0.22 & 0.14 & 0.25 \\
\hline Platelets & -0.09 & 0.35 & -0.09 & 0.35 & -0.03 & 0.65 & -0.08 & 0.75 & -0.07 & 0.70 \\
\hline AFP & -0.03 & 0.43 & -0.17 & 0.40 & 0.45 & 0.03 & 0.15 & 0.40 & 0.12 & 0.30 \\
\hline HB & 0.14 & 0.52 & 0.18 & 0.52 & 0.14 & 0.30 & 0.10 & 0.39 & 0.15 & 0.29 \\
\hline WBCs & 0.15 & 0.16 & 0.04 & 0.38 & 0.17 & 0.50 & 0.19 & 0.20 & 0.09 & 0.70 \\
\hline Age & 0.21 & 0.23 & 0.02 & 0.78 & 0.19 & 0.70 & 0.07 & 0.80 & 0.03 & 0.90 \\
\hline BMI & -0.17 & 0.40 & -0.01 & 0.80 & 0.22 & 0.15 & 0.24 & 0.11 & 0.09 & 0.81 \\
\hline Lower limb edema & - & - & 0.39 & 0.03 & 0.06 & 0.56 & - & - & - & - \\
\hline
\end{tabular}

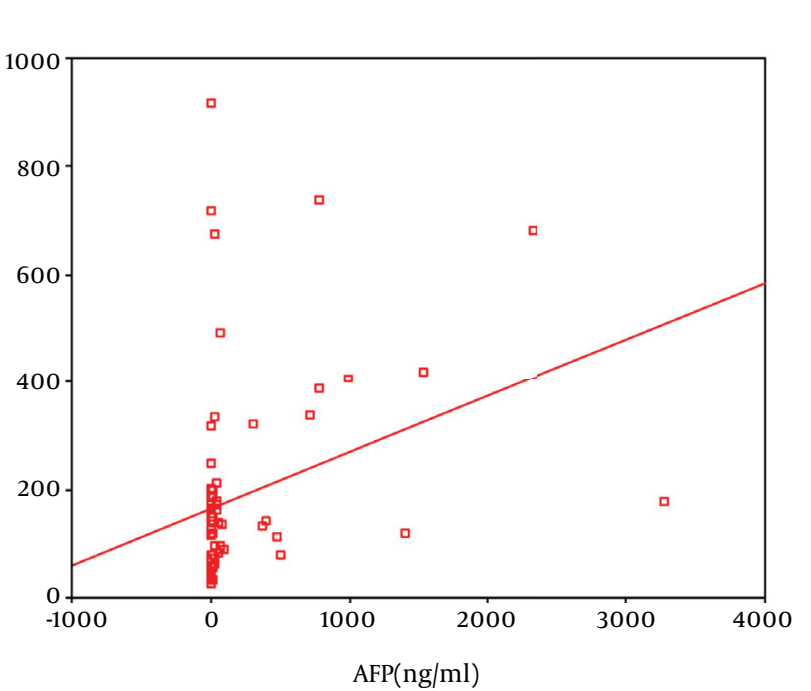

Figure 1. Correlation Between Plasma Osteopontin (OPN) and Alpha Fetoprotein (AFP) Levels in All Studied Groups, Showing a Significant Progressive Correlation as Serum AFP Level Increases With Increase in OPN Level $(\mathrm{P}<0.05)$

The mean plasma OPN level in the non-cirrhotic HCV group was $168.7 \pm 41 \mathrm{ng} / \mathrm{mL}$. The mean plasma OPN level was $258.3 \pm 35 \mathrm{ng} / \mathrm{mL}$ in the cirrhotic group and $401 \pm 72$ $\mathrm{ng} / \mathrm{mL}$ in the HCC group. Additionally, the level was 106.7 $\pm 35 \mathrm{ng} / \mathrm{mL}$ in the fatty liver group and $106.7 \pm 35 \mathrm{ng} / \mathrm{mL}$ in the control group. Our results showed that the highest level of serum OPN level was detected in the HCC group, followed by the cirrhotic group. According to the oneway ANOVA, there was a statistically significant difference in the serum OPN level between the control group and the different groups of patients. Also, a statistically significant difference in the serum OPN level was reported between the HCC group and the other groups. However, there was no significant difference in the OPN level between the patients with HCV without cirrhosis (Group I) and those with fatty liver (Group IV) (Table 3).

There was a statistically significant difference in the serum OPN level between the HCC group, the benign chronic liver disease groups (Groups I, II, and IV), and the control group $(\mathrm{P}=0.00)$ (Table 4$)$.

In the HCC group, the diagnostic value of OPN was comparable to that of AFP at a cutoff value of $280 \mathrm{ng} / \mathrm{mL}$, achieving sensitivity, specificity, and overall accuracy of $100 \%, 98 \%$, and $96 \%$, respectively. In contrast, in the cirrhotic group, OPN superseded AFP by achieving 93\%, 60\%, and $71 \%$ for sensitivity, specificity, and overall accuracy, correspondingly (Table 5 and Figure 2).

Regarding the validity of plasma OPN as a predictor of fatty change, our results revealed an AUC of $0.45 \%$ at a cutoff value of $134 \mathrm{ng} / \mathrm{mL}$, which showed $70 \%$ sensitivity, $45 \%$ specificity, $50 \%$ positive predictive value (PPV), and $75 \%$ negative predictive value (NPV) with accuracy of $50 \%$ (Table 5 and Figure 3 ). 
Fouad SA et al.

Table 3. Mean Plasma Osteopontin Level in Studied Groups

\begin{tabular}{lcccccc}
\hline Plasma Osteopontin Level & Group I $^{\mathbf{a}}$ & Group II $^{\mathbf{a}}$ & Group III $^{\mathbf{a}}$ & Group IV $^{\mathbf{a}}$ & Group V $^{\mathbf{b}}$ & PValues $^{\mathbf{c}}$ \\
\hline Mean \pm SD, ng/mL & $168.7 \pm 41$ & $258.3 \pm 35$ & $401 \pm 72$ & $106.7 \pm 35$ & $35.1 \pm 6$ & 0.001 \\
Range, $\mathbf{n g} / \mathbf{m L}$ & $95-224$ & $184-301$ & $287-494$ & $59-157$ & $25-44.9$ & 0.001 \\
\hline
\end{tabular}

$\mathrm{a}_{\mathrm{n}=25 \text {. }}$

$\mathrm{b} \mathrm{n}=20$.

C P values $<0.05$ are considered statistically significant. There was no significant difference between Group I and Group IV.

Table 4. Mean Plasma Osteopontin Level in Benign Chronic Liver Disease, Hepatocellular Carcinoma, and Control Groups

\begin{tabular}{lcccc}
\hline Plasma Osteopontin Level & $\begin{array}{c}\text { Benign Chronic Liver Disease } \\
\text { Groups (Groups I, II, \& IV) }(\mathbf{n}=\mathbf{7 5})\end{array}$ & $\begin{array}{c}\text { Hepatocellular Carcinoma } \\
\text { Group (Group III) }(\mathbf{n}=\mathbf{2 5})\end{array}$ & $\begin{array}{c}\text { Control Group } \\
(\text { Group V) (n= 20) }\end{array}$ & $\begin{array}{c}\text { P Values } \\
\mathbf{a}\end{array}$ \\
\hline Mean \pm SD, ng/mL & $177 \pm 60$ & $401 \pm 72$ & $35.1 \pm 6$ & 0.001 \\
Range, ng/mL & $59-224$ & $287-494$ & $25-44.9$ & 0.001 \\
\hline
\end{tabular}

a P values $<0.05$ are considered statistically significant.

Table 5. Validity of Osteopontin and Alpha Fetoprotein in the Prediction of Hepatocellular Carcinoma, Cirrhosis, and Nonalcoholic Fatty Liver Disease ${ }^{\text {a }}$

\begin{tabular}{lcccccccc}
\hline Variables & \multicolumn{2}{c}{ HCC } & \multicolumn{2}{c}{ Cirrhosis } & \multicolumn{2}{c}{ NAFLD } & \multicolumn{2}{c}{ Normal } \\
\cline { 2 - 10 } & OPN & AFP & OPN & AFP & OPN & AFP & OPN & AFP \\
\hline Best cutoff, ng/mL & 280 & 142 & 180 & 4 & 134 & 6.5 & 53 & 6 \\
AUC & 0.99 & 1.0 & 0.70 & 0.50 & 0.45 & 0.44 & 0.100 & 0.47 \\
Sensitivity, \% & 100 & 100 & 93 & 23 & 70 & 53 & 100 & 54 \\
\hline Specificity, \% & 98 & 100 & 60 & 40 & 45 & 60 & 100 & 57 \\
PPV,\% & 99 & 100 & 70 & 43 & 50 & 50 & 100 & 60 \\
NPV,\% & 100 & 100 & 94 & 44 & 75 & 55 & 100 & 55 \\
Accuracy, \% & 96 & 100 & 71 & 31 & 50 & 55 & 100 & 54 \\
\hline
\end{tabular}

a Abbreviations: AFP, $\alpha$-fetoprotein; AUC, area under the curve; HCC, hepatocellular carcinoma; NAFLD, nonalcoholic fatty liver disease; NPV, negative predictive value; OPN, osteopontin; $\mathrm{PPV}$, positive predictive value.

Figure 2. Receiver Operating Characteristic (ROC) Curve for Osteopontin (OPN) and Alpha Fetoprotein (AFP) in the Diagnosis of Hepatocellular Carcinoma

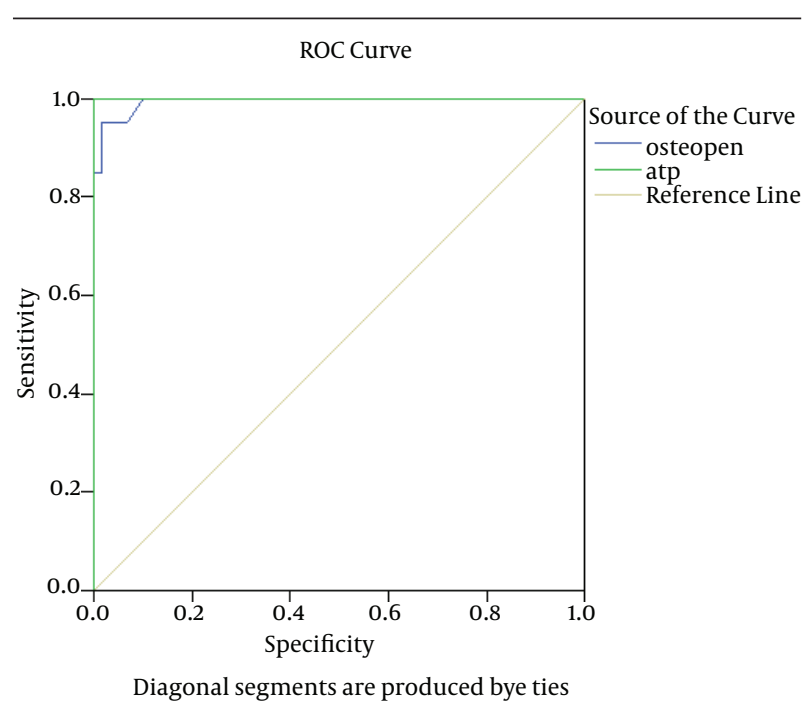

The mean AUC was 0.99 for OPN and 1.0 for AFP.
Figure 3. Receiver Operating Characteristic (ROC) Curve for Osteopontin (OPN) and Alpha Fetoprotein (AFP) in the Diagnosis of Nonalcoholic Fatty Liver Disease

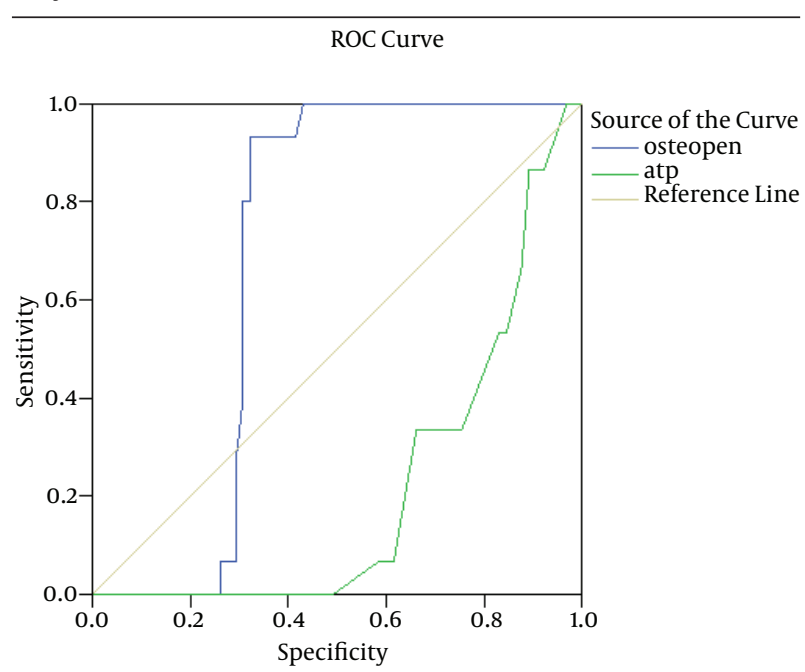

Diagonal segments are produced bye ties

The mean AUC was 0.45 for OPN and 0.44 for AFP. 


\section{Discussion}

The development of novel diagnostic markers for HCC has always been a significant pursuit of clinicians. The prognosis of HCC is serious with a great need for serum markers to start a therapeutic procedure at a potentially curable phase (16). Currently, AFP is the most validated serological marker for HCC, even though its performance in HCC is deficient (17). In fact, the only biochemical marker, AFP, is notoriously insufficient to detect a substantial proportion of HCC cases. Moreover, according to the practice guideline for HCC by the American Association for the Study of Liver Diseases (AASLD), AFP alone should not be used for HCC screening unless ultrasound is not available (14). Recent systematic reviews show that the quality of evidence supporting the use of AFP as a diagnostic and screening test for HCC is limited (18).

OPN, a protein encoded by phosphoprotein 1, a gene located in the same region as AFP (4q22.1 and 4q13, respectively, $14 \mathrm{Mb}$ apart), was first proposed as a prognostic marker of tumor recurrence and metastasis by some investigators about a decade ago (19). Since then, it has emerged that OPN levels rise in a variety of tumor and inflammatory processes, affecting various organs and tissues (20). More recently, OPN was proposed as a novel diagnostic marker to detect liver tumorigenesis. In the liver, it has been reported that hepatic Kupffer cells secrete OPN, which facilitates macrophage infiltration into necrotic areas following carbon tetrachloride toxicity (21). OPN is an attractive potential tumor marker because it exists not only as an immobilized extracellular matrix molecule but also in a secreted form in body fluids such as plasma (22). The role of OPN in HCC has also generated a significant interest, especially with regard to its role as a prognostic factor. Moreover, recent work has highlighted the role of OPN in inflammatory liver diseases such as alcoholic and nonalcoholic disease and T-cell mediated hepatitis (23).

Our work aims to create more interest in OPN inclusion in the diagnostic process of HCC in Egyptian patients. Moreover, we sought to extend the diagnostic impact of OPN to fatty liver disease, a significant condition reported in the Egyptian population. Consequently, we tried to validate the serum OPN level in HCV patients with and without cirrhosis, HCC patients on top of chronic HCV with cirrhosis, and NAFLD patients with a view to verifying the possibility of using the serum OPN level as a potential biomarker for HCC and as a disease predictor in NAFLD.

In concordance with some other reports $(24,25)$, we found that the serum level of AFP was significantly higher in the HCC patients (Group III) than in the other groups, including the cirrhotic group $(\mathrm{P}<0.05)$. The sensitivity and specificity of the serum AFP level in HCC detection has been shown to vary with the different cutoff values used. According to our results, at a cutoff $142 \mathrm{ng} / \mathrm{mL}$, both the sensitivity and the specificity were $100 \%$.

With respect to liver cirrhosis prediction, our study revealed that the sensitivity and specificity of AFP were $23 \%$ and $40 \%$, with $43 \%$ PPV and 44\% NPV. Moreover, the sensitivity and specificity of AFP in fatty change prediction were $53 \%$ and $60 \%$, respectively, with 50\% PPV and 55\% NPV. We reported a significant inverse correlation between AFP and the platelet count as the patients with a low platelet count had higher levels of serum AFP. This may be explained by the progression of liver cirrhosis with a subsequent decreased platelet count (due to portal hypertension and splenomegaly).

To our knowledge, our study is one of the earliest studies to describe the predictive value of OPN in Egyptian patients with chronic liver disease and HCC. Comparing the median plasma OPN level between the different groups, we found a significant elevation in the OPN level in the HCC patients $(401 \mathrm{ng} / \mathrm{mL}$ ) and the cirrhotic HCV patients (258.3 ng/mL) compared to the normal control group (35.1 $\mathrm{ng} / \mathrm{mL}$ ). Additionally, the plasma OPN level was higher in the cirrhotic HCV patients than in the non-cirrhotic HCV group (168.7 ng/mL) and the fatty liver group (106 ng/mL). However, argument of such values was reported by other researchers (19, 22, 26-28).

Plasma OPN proved to have diagnostic accuracy in the prediction of cirrhotic liver disease. At a cutoff value of $180 \mathrm{ng} / \mathrm{mL}$, it showed 93\% sensitivity, 60\% specificity, 70\% PPV, and 94\% NPV with overall accuracy of $71 \%$.

NAFLD has become the most prevalent cause of liver disease in Western countries. It includes a spectrum of diseases ranging from simple steatosis to inflammatory NASH, with increasing levels of fibrosis and ultimately cirrhosis. The diagnosis of NASH can be confirmed only by liver biopsy (28). In an attempt to draw attention to the possible role of plasma OPN as a predictor of fatty change in the liver, we reported that the plasma OPN level was higher in the patients with NAFLD than in the controls $(\mathrm{P}<0.05)$. At a cutoff value of $134 \mathrm{ng} / \mathrm{mL}$, it showed sensitivity of $70 \%$, specificity of $45 \%$, PPV of $50 \%$, and NPV of $75 \%$ with overall accuracy of $50 \%$. Our results proved that OPN is sensitive in the detection of fatty change in the liver; it could, therefore, be used as a simple, noninvasive, low-cost method for the screening and early identification of NAFLD.

Regarding the diagnostic value of OPN in HCC, pioneering surveys conducted by Egyptian investigators (29-31) reported that plasma OPN could be considered a potential diagnostic biomarker for HCC in Egyptian patients with HCV. However, the authors reported different cutoff values of OPN levels (ranging from 9.3 to $300 \mathrm{ng} / \mathrm{mL}$ ), which may be explained by the differences in the sample size, the study population, and the kit used to detect OPN levels. Further studies are necessary to fully explain such discrepancies.

In our study, the sensitivity, specificity, PPV, and NPV of OPN for the selective detection of the HCC group over the benign chronic liver disease groups were 100\%, 98\%, 99\%, and $100 \%$, correspondingly, at a cutoff level of $280 \mathrm{ng} / \mathrm{mL}$ with overall accuracy of $96 \%$. 
Fouad SA et al.

Our results confirm those by some previous studies (27) reporting that the diagnostic efficacy of OPN is comparable to that of AFP with a significant positive correlation between OPN and AFP levels in terms of sensitivity, specificity, PPV, and NPV. Nonetheless, some studies have found that the correlation between plasma OPN and serum AFP levels is insignificant $(22,31)$.

The sensitivity and specificity of OPN in HCC have been shown to vary with different cutoff values. Moreover, the reported normal median plasma OPN levels are highly variable, ranging from $31 \mathrm{ng} / \mathrm{mL}$ to even greater than 200 $\mathrm{ng} / \mathrm{mL}$, as reported in the literature $(19,22,32)$. However, Matsui et al suggested that $200 \mathrm{ng} / \mathrm{mL}$ could be set as the critical cutoff point for predicting patients with HCC (20). The exact reason for these differences is not clear, but these discrepancies may be in consequence of the different assay systems and conditions of sample collection used in different studies.

Collectively, our results indicated that plasma OPN could be used in the selective detection and diagnosis of HCC. Also, OPN was superior to AFP in predicting liver cirrhosis and fatty change, which are risk factors for HCC.

In conclusion, the results of the current study revealed that the plasma OPN level was elevated in the HCV-related HCC patients by comparison to the benign cirrhotic and non-cirrhotic HCV and NAFLD patients. Notably, our data demonstrated that the plasma OPN level was elevated in NAFLD, which could be related to fatty liver change by reflecting OPN overexpression in the hepatic parenchyma. Thus, OPN represents a potential biomarker for the detection of NAFLD. Also, OPN is relatively comparable to AFP in the detection of HCC among high-risk groups and is superior to AFP in the overall prediction of chronic liver disease. Although AFP has been considered the golden standard serum marker for HCC for years, in light of our data, the usefulness of AFP testing as the only biomarker for the population at risk should be questioned.

\section{Acknowledgements}

We acknowledge the Internal Medicine Department, Faculty of Medicine, Cairo University.

\section{Authors' Contributions}

Shawky Abdelhamid Fouad proposed the research idea and design, assessed the patients, collected and analyzed the data, and wrote the manuscript. Nagwa Abdel Ghaffar Mohamed carried out the laboratory part of the research. Mary Wadie Fawzy contributed to the design of the research. All authors read and approved the manuscript.

\section{References}

1. Nagoshi S. Osteopontin: Versatile modulator of liver diseases. Hepatol Res. 2014;44(1):22-30.

2. Ashkar S, Weber GF, Panoutsakopoulou V, Sanchirico ME, Jansson M, Zawaideh S, et al. Eta-1 (osteopontin): an early component of type-1 (cell-mediated) immunity. Science. 2000;287(5454):860-4.

3. Suzuki H, Amizuka N, Oda K, Li M, Yoshie H, Ohshima H, et al. His- tological evidence of the altered distribution of osteocytes and bone matrix synthesis in klotho-deficient mice. Arch Histol Cytol. 2005;68(5):371-81.

4. Denhardt DT, Guo X. Osteopontin: a protein with diverse functions. FASEB J.1993;7(15):1475-82.

5. Huang W, Zhu G, Huang M, Lou G, Liu Y, Wang S. Plasma osteopontin concentration correlates with the severity of hepatic fibrosis and inflammation in HCV-infected subjects. Clin Chim Acta. 2010;411(9-10):675-8.

6. Wu CY, Wu MS, Chiang EP, Wu CC, Chen YJ, Chen CJ, et al. Elevated plasma osteopontin associated with gastric cancer development, invasion and survival. Gut. 2007;56(6):782-9.

7. Syn WK, Choi SS, Liaskou E, Karaca GF, Agboola KM, Oo YH, et al. Osteopontin is induced by hedgehog pathway activation and promotes fibrosis progression in nonalcoholic steatohepatitis. Hepatology. 2011;53(1):106-15.

8. Ranjbar Kermani F, Sharifi Z, Ferdowsian F, Paz Z, Tavassoli F. The Usefulness of Anti-HCV Signal to Cut-off Ratio in Predicting Viremia in Anti-HCV in Patients With Hepatitis C Virus Infection. Jundishapur J Microbiol. 2015;8(4):e17841.

9. Moini M, Ziyaeyan M, Aghaei S, Sagheb MM, Taghavi SA, Moeini M, et al. Hepatitis C virus (HCV) Infection Rate among Seronegative Hemodialysis Patients Screened by Two Methods; HCV Core Antigen and Polymerase Chain Reaction. Hepat Mon. 2013;13(6):e9147.

10. Parkin DM, Bray F, Ferlay J, Pisani P. Estimating the world cancer burden: Globocan 2000. Int J Cancer. 2001;94(2):153-6.

11. Charlton M. Hepatitis C infection in liver transplantation. Am J Transplant. 2001;1(3):197-203.

12. El-Zanaty F, Wany A, Macro International.. Egypt Demographic and Health Survey 2008.Cairo: Egyptian: Ministry of Health;2009.

13. Lavanchy D. Evolving epidemiology of hepatitis C virus. Clin Microbiol Infect. 2011;17(2):107-15

14. Bruix J, Sherman M, Practice Guidelines Committee AAFTSOLD. Management of hepatocellular carcinoma. Hepatology. 2005;42(5):1208-36.

15. Ostheimer C, Bache M, Guttler A, Reese T, Vordermark D. Prognostic information of serial plasma osteopontin measurement in radiotherapy of non-small-cell lung cancer. BMC Cancer. 2014;14:858.

16. Fouad SA, Elsaaid NH, Mohamed NA, Abutaleb OM. Diagnostic Value of Serum Level of Soluble Tumor Necrosis Factor Receptor IIalpha in Egyptian Patients With Chronic Hepatitis C Virus Infection and Hepatocellular Carcinoma. Hepat Mon. 2014;14(9):e19346.

17. Lok AS, Gardiner DF, Lawitz E, Martorell C, Everson GT, Ghalib R, et al. Preliminary study of two antiviral agents for hepatitis $C$ genotype 1. NEngl J Med. 2012;366(3):216-24.

18. Colli A, Fraquelli M, Conte D. Alpha-fetoprotein and hepatocellular carcinoma. Am J Gastroenterol . 2006;101(8):1939.

19. Shang S, Plymoth A, Ge S, Feng Z, Rosen HR, Sangrajrang S, et al Identification of osteopontin as a novel marker for early hepatocellular carcinoma. Hepatology. 2012;55(2):483-90.

20. Matsui A, Mochida S, Ohno A, Nagoshi S, Hirose T, Fujiwara K. Plasma osteopontin levels in patients with fulminant hepatitis. Hepatol Res. 2004;29(4):202-6.

21. Wang JH, Changchien CS, Hu TH, Lee CM, Kee KM, Lin CY, et al. The efficacy of treatment schedules according to Barcelona Clinic Liver Cancer staging for hepatocellular carcinoma - Survival analysis of 3892 patients. Eur J Cancer. 2008;44(7):1000-6.

22. Kim J, Ki SS, Lee SD, Han CJ, Kim YC, Park SH, et al. Elevated plasma osteopontin levels in patients with hepatocellular carcinoma. Am J Gastroenterol. 2006;101(9):2051-9.

23. Ramaiah SK, Rittling S. Pathophysiological role of osteopontin in hepatic inflammation, toxicity, and cancer. Toxicol Sci. 2008;103(1):4-13.

24. Taketa K, Okada S, Win N, Hlaing NK, Wind KM. Evaluation of tumor markers for the detection of hepatocellular carcinoma in Yangon General Hospital, Myanmar. Acta Med Okayama. 2002;56(6):317-20.

25. Gad A, Tanaka E, Matsumoto A, El-Hamid Serwah A, Attia F, Hassan A, et al. Ethnicity affects the diagnostic validity of alpha-fetoprotein in hepatocellular carcinoma. Asia-Pacific J Clin Oncol. 2005;1(2-3):64-70. 
26. Zhao L, Li T, Wang Y, Pan Y, Ning H, Hui X, et al. Elevated plasma osteopontin level is predictive of cirrhosis in patients with hepatitis B infection. Int JClin Pract. 2008;62(7):1056-62.

27. Zhang CH, Xu GL, Jia WD, Ge YS, Li JS, Ma JL, et al. Prognostic significance of osteopontin in hepatocellular carcinoma: a metaanalysis. Int J Cancer. 2012;130(11):2685-92.

28. Yilmaz Y, Ozturk O, Alahdab YO, Senates E, Colak Y, Doganay HL, et al. Serum osteopontin levels as a predictor of portal inflammation in patients with nonalcoholic fatty liver disease. Dig Liver Dis. 2013;45(1):58-62.

29. El-Din Bessa SS, Elwan NM, Suliman GA, El-Shourbagy SH. Clinical significance of plasma osteopontin level in Egyptian patients with hepatitis C virus-related hepatocellular carcinoma. Arch Med Res. 2010;41(7):541-7.

30. Abu El Makarem MA, Abdel-Aleem A, Ali A, Saber R, Shatat M, Rahem DA, et al. Diagnostic significance of plasma osteopontin in hepatitis C virus-related hepatocellular carcinoma. Ann Hepatol. 2011;10(3):296-305.

31. Nabih MI, Aref WM, Fathy MM. Significance of plasma osteopontin in diagnosis of hepatitis $C$ virus-related hepatocellular carcinoma. Arab J Gastroenterol. 2014;15(3-4):103-7.

32. Keddeas MW, Abo-shady RA. Evaluation of plasma osteopontin level as a biomarker for hepatocellular carcinoma in Egyptian patients. Egypt Liver J. 2011;1(1):38-42. 